\title{
The Orbit of the Visual Binary ADS 8630
}

\author{
Marco Scardia ${ }^{1}$, Robert Argyle ${ }^{2}$, Jean-Louis Prieur ${ }^{3}$, \\ Luigi Pansecchi ${ }^{1}$, Stefano Basso ${ }^{1}$, Nicholas Law $^{2}$ and Craig Mackay ${ }^{2}$ \\ ${ }^{1}$ I.N.A.F. - Osservatorio Astronomico di Brera, Via E. Bianchi 46, 23807 Merate, Italy \\ ${ }^{2}$ Institute of Astronomy, Madingley Road, Cambridge, CB3 0HA, UK \\ email: rwa@ast.cam.ac.uk \\ ${ }^{3}$ UMR 5572 d'Astrophysique, Observatoire Midi-Pyrénées, CNRS, Univ. Paul Sabatier, 14, \\ Avenue Edouard Belin, 31400 Toulouse, France
}

\begin{abstract}
.
Using the 35 speckle measurements performed with PISCO in Merate between 2004 and 2006, and some complementary new astrometric measures, we present a new orbit for the visual binary ADS $8630=\gamma$ Vir. This new series of measurements was essential for constraining the 169 yearperiod orbit since it covers an arc of $130^{\circ}$ in the apparent orbit and includes the periastron passage of 2005 .
\end{abstract}

Keywords. Stars: binaries: close, visual - Astrometry - Techniques: interferometric

\section{Introduction}

Gamma Virginis (STF1670 = WDS 12417-0127) is one of the most closely followed of visual binaries. The WDS observations catalogue (2006 June) contains 1515 mean measures of position angle and separation. Despite this, the very high eccentricity of the orbit and the difficulty of observing the pair at periastron passage in 1836 has meant that it is only now that sufficent measures of the recent close approach in 2005 and the period immediately after, which were badly underobserved in 1836 and 1837, have allowed an orbital analysis which predicts the angular motion to an acceptable degree of accuracy.

\section{The orbit}

In addition to the measures available in the WDS observations catalogue, we use measurements made by ourselves during mid-2004, the periastron passage of Summer 2005 (Scardia et al. 2006a) and in mid-2006, and also by Dr. A. Alzner (Alzner, 2006) in mid- and late-2005. A total of 1553 measurements was used for the first iteration.

Each observation has been corrected for precession, proper motion and radial velocity according to the relation outlined by Fletcher (1931). Although these corrections are small, they are necessary in the derivation of a definitive orbit. Whilst making the last differential correction to the orbit, we rejected those observations with position angle residuals greater than $4^{\circ} .2(3 \sigma)$ and separations with residuals greater than $0^{\prime \prime} .2(1.7 \sigma)$.

The scatter in position angle is surprisingly small, considering the range of epoch over which the observations were made (1718 to 2006) and the large number of observers using every type of telescope, the apertures of which range from $10-\mathrm{cm}$ to $400-\mathrm{cm}$. However the scatter in separation is much larger, possibly due to the great brightness of the two stars which hinders accurate measurement with the micrometer.

Thirty-five of the most recent measures were made by ourselves with the PISCO camera 


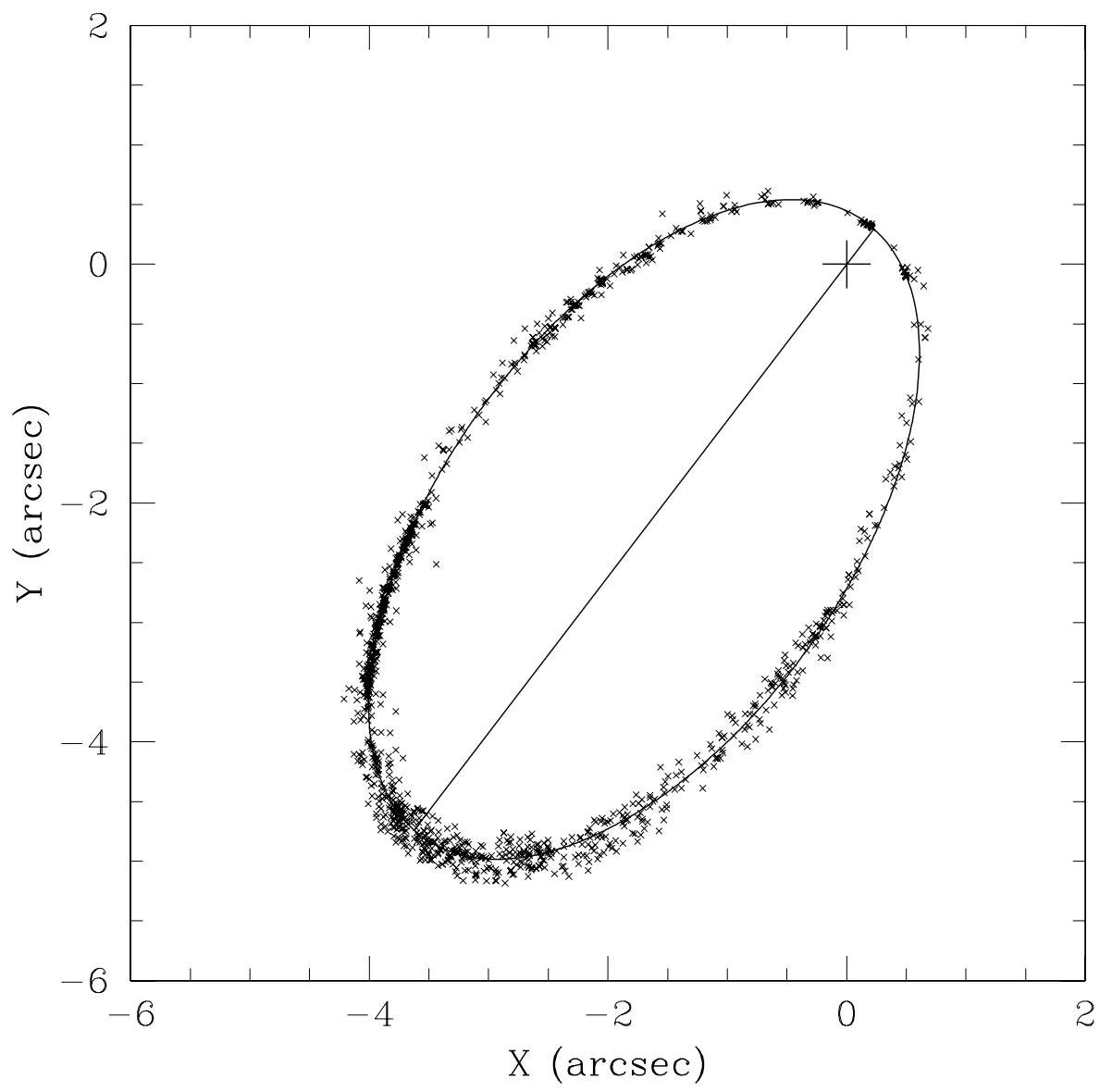

Figure 1. The apparent orbit of $\gamma$ Virginis

on the 102-cm Zeiss telescope at the I.N.A.F., Osservatorio Astronomico di Brera in Merate. Details of the instrument can be found elsewhere (Prieur et al. 1998). Particular care has been taken with the calibration of scale and orientation in the plane of the detector by the use of an objective grating (Scardia et al. 2006a). All of the PISCO measures were made with a $10-\mathrm{mm}$ eyepiece, yielding an image scale of $0^{\prime \prime} .0320 \pm 0^{\prime \prime} .0001$ per pixel.

The 2004-2006 PISCO observations cover an arc of $130^{\circ}$ and the apparent orbit is shown in Figure 1, with an expanded view in Figure 2. A recent image of the system taken with the LuckyCam detector on the 3.6-m NTT in Chile (Law, 2006) is shown in Figure 7 .

The orbital elements that we derived are shown in Table 1. Residuals from this orbit are given in Table 2 for the observations made since the beginning of 2000 . The ephemeris from these orbital elements for the years 2006-2015 is given in Table 3. The Hipparcos parallax is $0^{\prime \prime} .085 \pm 0^{\prime \prime} .001$ and the sum of the masses is $2.8 \pm 0.1 \mathrm{M}_{\odot}$; the semi-major axis is $43.0 \mathrm{AU}$. The dynamical parallax as derived from the Baize-Romani relationship (Baize \& Romani, 1945) is $0^{\prime \prime} .0824$, with an estimated error of $4 \%$. It is difficult to evaluate an error for this quantity as this method uses the spectral types in a tabulated form. 


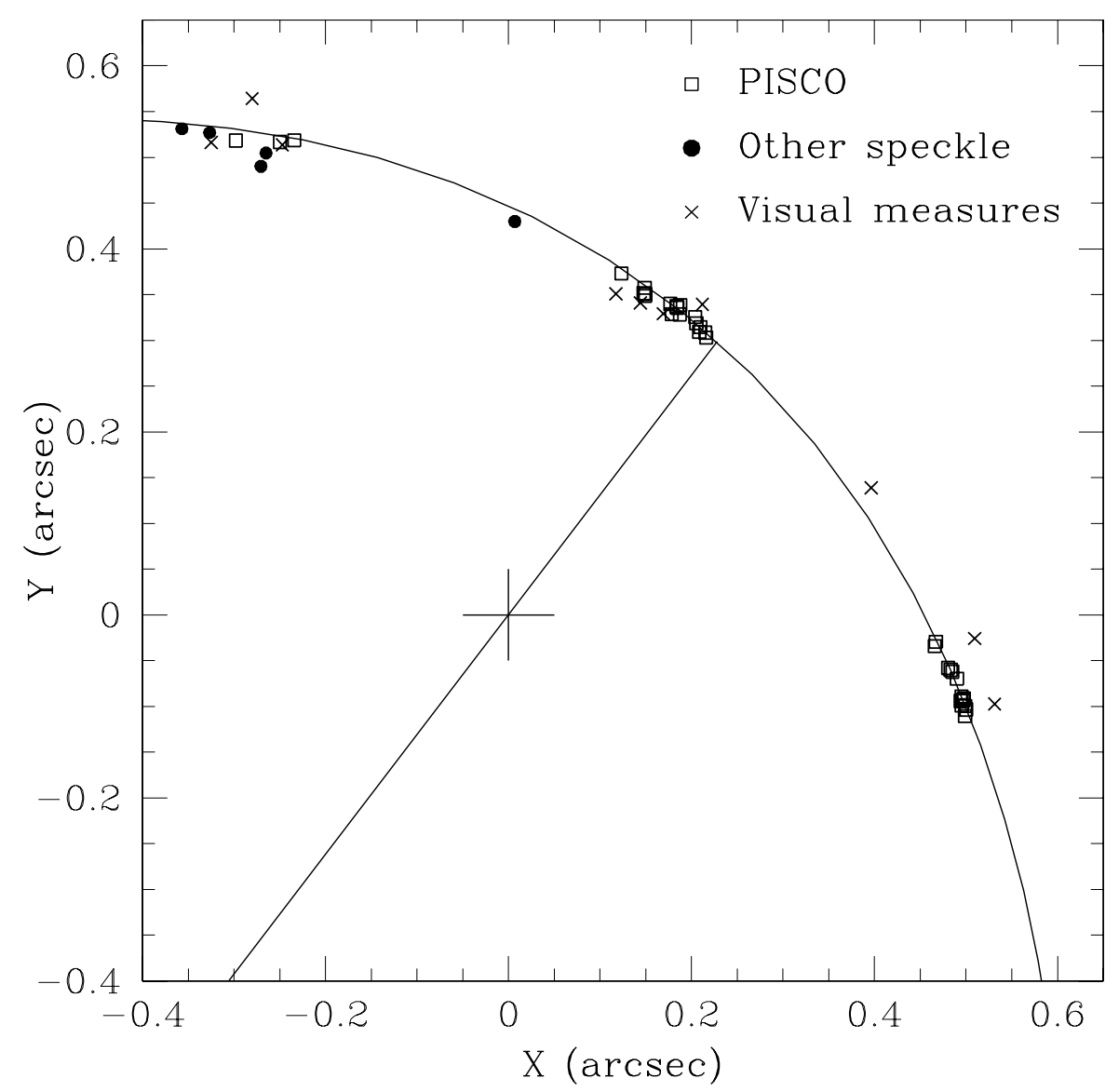

Figure 2. Enlarged view of Figure 1 around periastron

Table 1. New orbital elements of ADS 8630

\begin{tabular}{|c|c|c|c|c|c|c|c|c|c|c|}
\hline $\begin{array}{l}\text { Node } \\
2000\left(^{\circ}\right)\end{array}$ & $\begin{array}{c}\text { omega } \\
\left({ }^{\circ}\right)\end{array}$ & $\begin{array}{c}i \\
\left(^{\circ}\right)\end{array}$ & $e$ & $\begin{array}{c}T \\
(\mathrm{yr})\end{array}$ & $\begin{array}{c}P \\
(\mathrm{yr})\end{array}$ & $\begin{array}{c}a \\
\left({ }^{\prime \prime}\right)\end{array}$ & $\begin{array}{c}\mathrm{A} \\
\left({ }^{\prime \prime}\right)\end{array}$ & $\begin{array}{c}\mathrm{B} \\
\left({ }^{\prime \prime}\right)\end{array}$ & $\underset{\left({ }^{\prime \prime}\right)}{\mathrm{F}}$ & $\begin{array}{c}\mathrm{G} \\
\left({ }^{\prime \prime}\right)\end{array}$ \\
\hline $\begin{array}{l}35.34 \\
\pm 0.42\end{array}$ & $\begin{array}{c}255.02 \\
\pm 0.37\end{array}$ & $\begin{array}{c}149.46 \\
\pm 0.16\end{array}$ & $\begin{array}{c}0.8815 \\
\pm 0.00018\end{array}$ & $\begin{array}{c}2005.511 \\
\pm 0.0019\end{array}$ & $\begin{array}{l}169.104 \\
\pm 0.011\end{array}$ & $\begin{array}{c}3.639 \\
\pm 0.008\end{array}$ & -2.51886 & 1.92543 & 2.39886 & 2.69458 \\
\hline
\end{tabular}

The trigonometrical and dynamical parallaxes agree to within their respective errors, and the resulting Baize-Romani mass sum is $3.0 \pm 0.1 \mathrm{M}_{\odot}$, which agrees with the mass computed with the Hipparcos parallax to about $10 \%$.

\section{Residuals from the visual orbit}

We examined the observations which were deemed to be most accurate: the photographic measures taken mainly at the USNO from 1950-1975 and speckle measurements from 1980 onwards. Figures 3 and 4 show the residuals from the orbit for these obser- 


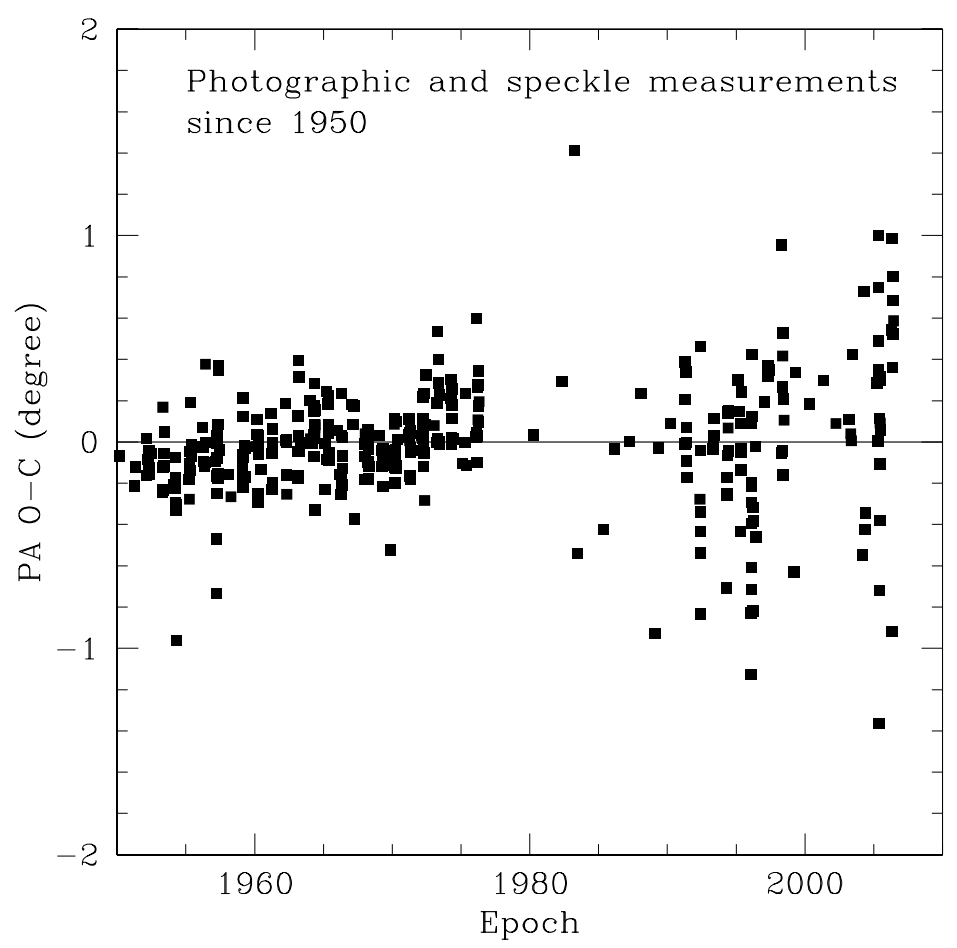

Figure 3. Orbital residuals in position angle 1950-2006

vations. The photographic results show no features with amplitude greater than about $0^{\prime \prime} .02$.

From 1994 to 2005 however, the speckle results do seem to show a systematic residual. Over this period the separation of the pair reduced from over $2^{\prime \prime}$ to less than $0^{\prime \prime} .4$. Figure 5 shows the residuals for the USNO speckle results only. There is clearly a small systematic offset in separation from the predicted orbital position. This agrees with the conclusions drawn by Germain et al. (1999) who note that '..pairs observed with the 10-power microscope objective show a 20 mas proximity effect for separations less than $1^{\prime \prime}$.5'. In other words, the observed separation is measured to be smaller than it really is. Germain \& Douglass (2001) explained the proximity effect as being due to incomplete removal of non-uniform background arising from seeing and from additive noise and it is relatively more significant for closer binaries. Background estimation used in the USNO speckle program applies a running boxcar filter to the average autocorrelation function but because the boxcar filter understimates the background, the proximity effect survives.

Alternatively, Brian Mason (private communication) suggested that the systematic effects seen in Figure 5 would disappear if a slightly smaller major axis was used. To test this hypothesis, a further orbital analysis was undertaken allocating greater weights to the USNO speckle observations, but this resulted in the USNO photographic data not fitting the calculated orbit quite as well, so the original set of orbital elements was not changed. 
Table 2. Orbital residuals of measurements from $2000-2006$

\begin{tabular}{llll}
\hline Epoch & $\rho(O)$ & $\begin{array}{l}\Delta \rho(O-C) \\
\left({ }^{\prime \prime}\right)\end{array}$ & $\begin{array}{l}\left(^{\prime \prime}\right) \\
\left({ }^{\circ}\right)\end{array}$ \\
\hline 2000.280 & 1.440 & -0.00 & -0.1 \\
2000.340 & 1.410 & -0.02 & +0.1 \\
2000.345 & 1.400 & -0.03 & -0.1 \\
2000.350 & 1.650 & +0.22 & -0.5 \\
2000.360 & 1.460 & +0.03 & -0.5 \\
2000.438 & 1.330 & -0.09 & +0.9 \\
2001.260 & 1.240 & -0.03 & +0.3 \\
2001.310 & 1.270 & +0.01 & -0.2 \\
2001.320 & 1.220 & -0.04 & +0.2 \\
2001.350 & 1.200 & -0.05 & -0.2 \\
2001.397 & 1.300 & +0.06 & -1.9 \\
2001.433 & 1.170 & -0.06 & -1.2 \\
2002.252 & 1.020 & -0.05 & +0.1 \\
2002.314 & 1.060 & +0.00 & -1.8 \\
2002.360 & 1.040 & -0.01 & +0.3 \\
2003.229 & 0.830 & -0.03 & +0.2 \\
2003.320 & 0.810 & -0.03 & +0.1 \\
2003.340 & 0.830 & -0.01 & +1.4 \\
2003.349 & 0.870 & +0.04 & +1.0 \\
2003.379 & 0.800 & -0.03 & +0.1 \\
2003.485 & 0.760 & -0.04 & +0.5 \\
2004.147 & 0.640 & -0.01 & +0.3 \\
2004.196 & 0.620 & -0.01 & -0.4 \\
2004.250 & 0.610 & -0.01 & +1.6 \\
2004.306 & $0.598^{P}$ & -0.01 & +1.2 \\
2004.309 & 0.570 & -0.04 & -1.1 \\
2004.333 & 0.630 & +0.03 & -1.6 \\
2004.365 & 0.560 & -0.03 & +1.9 \\
2004.380 & 0.570 & -0.02 & -0.8 \\
2004.397 & $0.572^{P}$ & -0.01 & +0.1 \\
2004.443 & $0.567^{P}$ & -0.00 & +0.1 \\
2004.998 & 0.430 & -0.01 & -0.0 \\
2005.228 & 0.370 & -0.03 & -2.8 \\
2005.259 & $0.392^{P}$ & -0.01 & -0.2 \\
2005.319 & $0.386^{P}$ & -0.01 & -0.1 \\
2005.326 & 0.370 & -0.02 & -0.1 \\
2005.328 & $0.380^{P}$ & -0.01 & +0.4 \\
2005.442 & $0.383^{P}$ & +0.00 & -0.1 \\
2005.330 & $0.380^{P}$ & -0.01 & +0.2 \\
2005.333 & $0.378^{P}$ & -0.01 & +0.4 \\
2005.382 & $0.373^{P}$ & -0.01 & $-1.2 \dagger$ \\
2005.385 & $0.382^{P}$ & -0.00 & +0.1 \\
2005.390 & 0.370 & -0.01 & +0.6 \\
2005.396 & $0.383^{P}$ & +0.00 & -0.2 \\
2005.398 & $0.382^{P}$ & -0.00 & -0.2 \\
2005.404 & $0.386^{P}$ & +0.00 & +0.0 \\
2005.409 & $0.377^{P}$ & -0.01 & -0.3 \\
2005.427 & 0.400 & +0.02 & -1.3 \\
\hline & $0.375^{P}$ & -0.00 & +0.1 \\
+005 & $0.378^{P}$ & -0.00 & -0.1 \\
\hline & & &
\end{tabular}

$P=$ PISCO observation; $\dagger=$ autocorrelation peaks were abnormally elongated for this observation 
Table 2. Orbital residuals of measurements from $2000-2006$, continued

\begin{tabular}{llll}
\hline Epoch & $\begin{array}{l}\rho(O) \\
\left({ }^{\prime \prime}\right)\end{array}$ & $\begin{array}{l}\Delta \rho(O-C) \\
\left(^{\prime \prime}\right)\end{array}$ & $\begin{array}{l}\Delta \theta(O-C) \\
\left({ }^{\circ}\right)\end{array}$ \\
\hline 2005.486 & $0.371^{P}$ & -0.01 & +0.1 \\
2005.964 & 0.420 & +0.02 & +2.3 \\
2006.273 & 0.510 & +0.05 & -0.7 \\
2006.308 & $0.466^{P}$ & -0.01 & +0.6 \\
2006.311 & $0.465^{P}$ & -0.01 & +0.2 \\
2006.360 & $0.482^{P}$ & -0.00 & -0.1 \\
2006.371 & $0.485^{P}$ & -0.00 & +0.2 \\
2006.376 & $0.487^{P}$ & -0.00 & +0.3 \\
2006.396 & 0.540 & +0.05 & -2.1 \\
2006.398 & $0.493^{P}$ & -0.00 & +0.5 \\
2006.439 & $0.501^{P}$ & -0.00 & +0.3 \\
2006.442 & $0.504^{P}$ & -0.00 & +0.3 \\
2006.447 & $0.504^{P}$ & -0.00 & +0.4 \\
2006.450 & $0.501^{P}$ & -0.01 & +0.2 \\
2006.453 & $0.505^{P}$ & -0.00 & +0.5 \\
2006.464 & $0.503^{P}$ & -0.01 & +0.3 \\
2006.475 & $0.508^{P}$ & -0.01 & +0.8 \\
2006.477 & $0.509^{P}$ & -0.01 & +0.5 \\
2006.497 & $0.509^{P}$ & -0.01 & +0.6 \\
\hline \multicolumn{5}{c}{$P$} & \multicolumn{2}{c}{ PISCO observation }
\end{tabular}

Table 3. New ephemeris of ADS 8630

\begin{tabular}{lcr}
\hline Epoch & $\begin{array}{c}\rho \\
\left({ }^{\prime \prime}\right)\end{array}$ & \multicolumn{1}{c}{$\theta$} \\
& $\left(^{\circ}\right)$ \\
\hline 2006.0 & 0.409 & 104.5 \\
2007.0 & 0.655 & 60.1 \\
2008.0 & 0.924 & 41.3 \\
2009.0 & 1.168 & 30.7 \\
2010.0 & 1.389 & 23.7 \\
2011.0 & 1.591 & 18.5 \\
2012.0 & 1.777 & 14.5 \\
2013.0 & 1.951 & 11.2 \\
2014.0 & 2.114 & 8.4 \\
2015.0 & 2.268 & 6.0 \\
\hline
\end{tabular}

\section{The mass of F0V stars}

The only other visual binary consisting of equally bright F0V stars and which has a reliable orbit is FIN 350 (Hartkopf, 1996) in which the masses were found to be $1.25 \mathrm{M}_{\odot}$ but with an uncertainty of $28 \%$. Mass-spectrum relationships in the literature suggest that the range of F0V masses is fairly large. Allen (1973) suggests a mass of $1.3 \mathrm{M}_{\odot}$, while Svechnikov (1984) give $1.46 \mathrm{M}_{\odot}$, Trimble (1974) gives $1.55 \mathrm{M}_{\odot}$, and Lang (1992) quotes Schmidt-Kaler giving 1.6 $\mathrm{M}_{\odot}$. Harmanec (1988), using an updated analysis of the data given by Popper (1980) found that for F0 main-sequence stars the range of mass is between 1.08 and $1.55 \mathrm{M}_{\odot}$ with an average of $1.33 \pm 0.10 \mathrm{M}_{\odot}$. The individual masses determined in this paper, i.e., $1.40 \pm 0.05 \mathrm{M}_{\odot}$, come close to both the average of the values from the work by Allen through to Schmidt-Kaler and also that of Harmanec.

Some previous studies have suggested the presence of a third companion (Herschel 


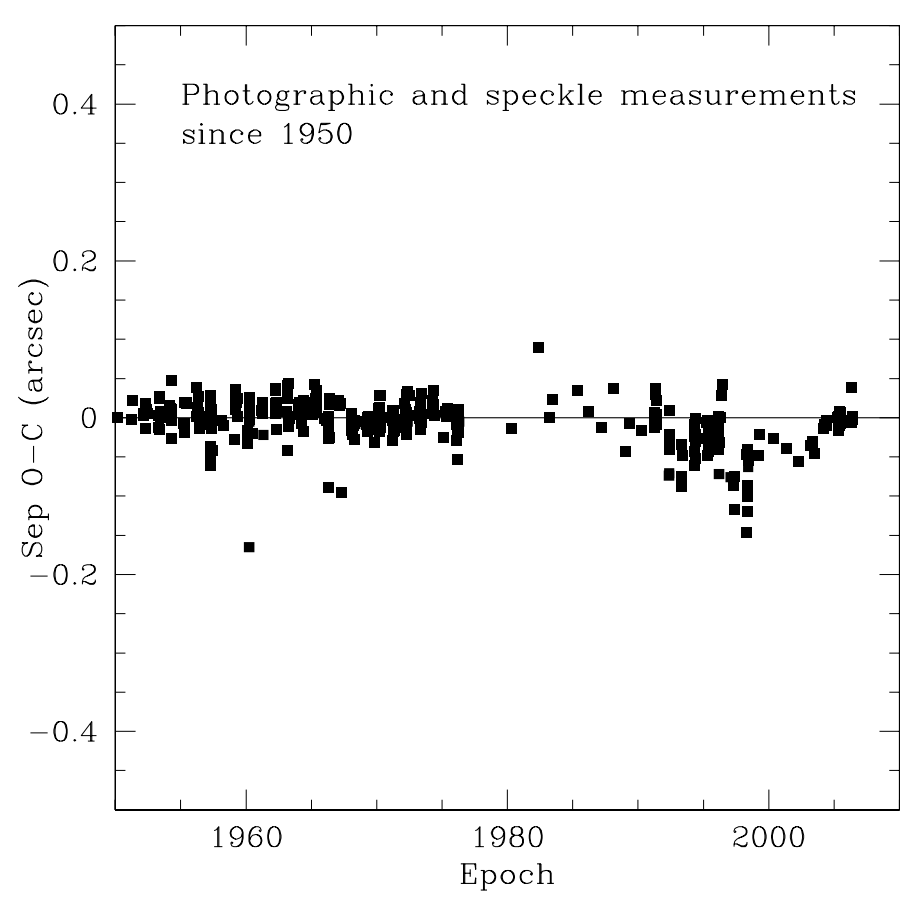

Figure 4. Orbital residuals in separation 1950-2006

1847). The mass ranges given above would suggest an upper limit for its mass at around $0.5 \mathrm{M}_{\odot}$. We discuss this possibility more fully in Scardia et al. (2006b).

\section{Conclusion}

The two-body orbit presented here gives acceptably small residuals for the critical part of the orbit through periastron. Hence, as a more detailed analysis has shown (Scardia et al., 2006b), the apparent orbit of ADS 8630 is fully compatible with a binary system and does not require the presence of a stellar third companion.

\section{Acknowledgements}

This research has made use of the Washington Double Star Catalog, maintained at the U.S. Naval Observatory. We thank Dr. B.D. Mason for discussions.

\section{References}

Allen, C. 1973, Astrophysical Quantities, 3rd edition, (Athlone Press) Alzner. A. 2006, Webb Society Double Star Section Circulars 14, 6. Baize, P. \& Romani, L. 1945, Ann. Astrophys. 9, 13

Fletcher, A. 1931, MNRAS 92, 119

Germain, M. E., Douglass, G. G, Worley, C. E. 1999, AJ 117, 2511

Germain, M. E. \& Douglass, G. G 2001, AJ 121, 2239

Harmanec, P. 1988, Bull. Astron. Inst. Czechosl. 39, 329

Hartkopf, W. I., Mason, B. D. \& McAlister, H. A. 1996, AJ 111, 370

Herschel, J. 1847, Results of astronomical observations made in the years 1834-1838 at the Cape of Good Hope, Smith, Elder \& Co., London 


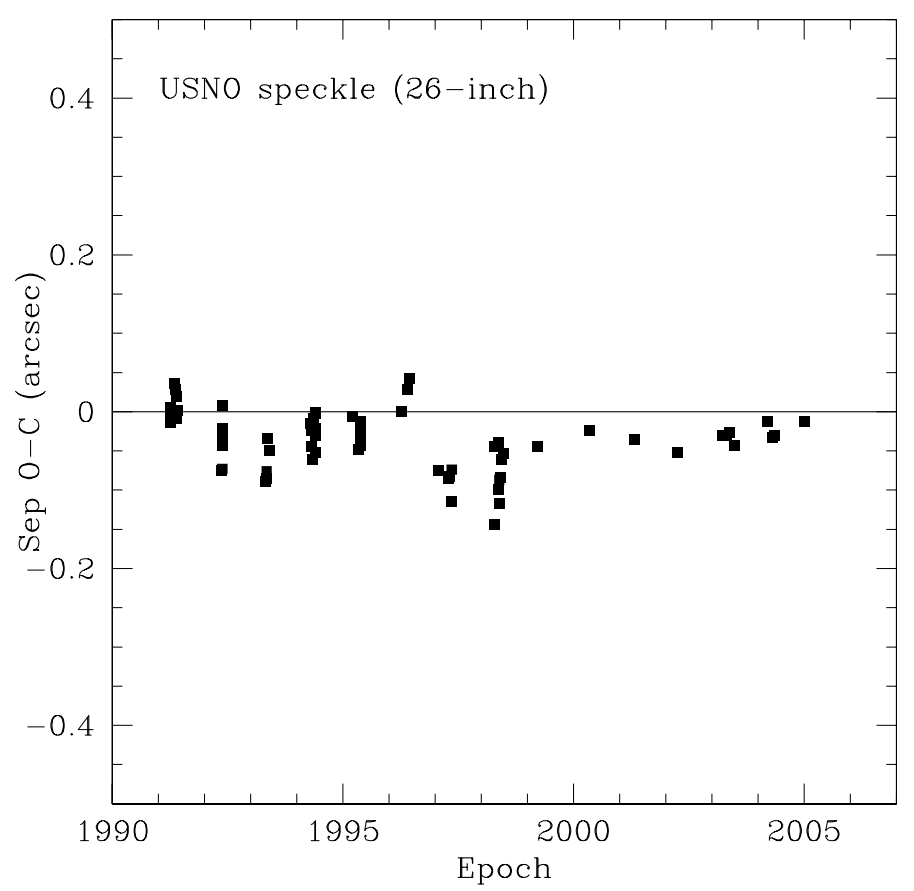

Figure 5. Orbital residuals in separation 1990-2005 (USNO speckle only)

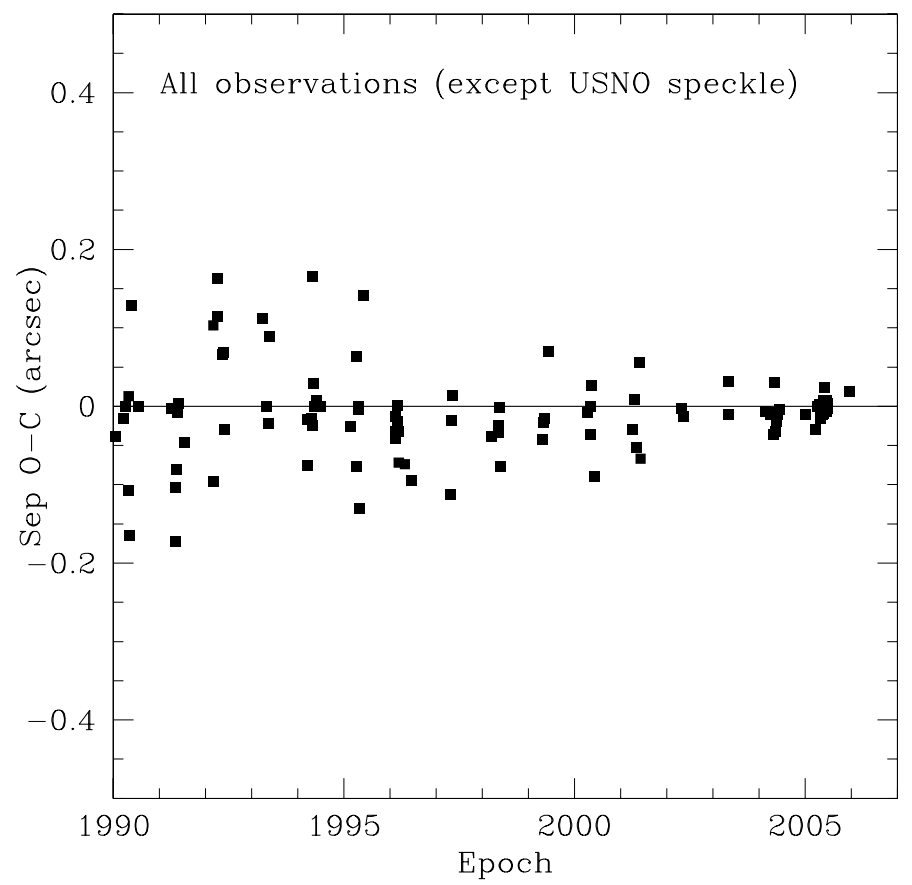

Figure 6. Orbital residuals in separation 1990-2005 (other than USNO speckle) 


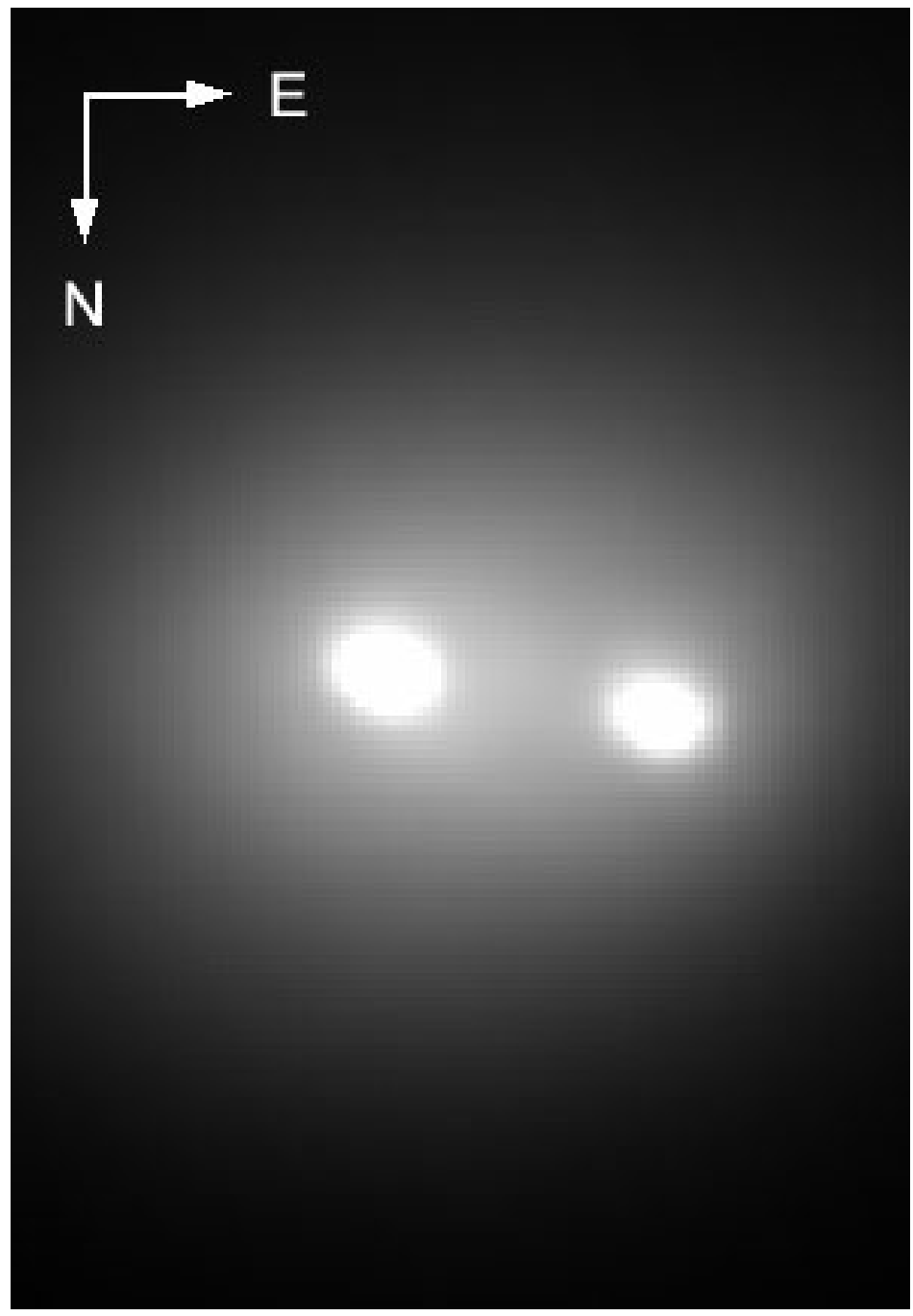

Figure 7. Unsharp-masked SDSS $i^{\prime}$-band image of $\gamma$ Vir A and B obtained with LuckyCam on the 3.6-m NTT at La Silla Observatory, Chile on 5 Jun 2006, when the angular separation was $0^{\prime \prime} .5$.

Lang, K 1992, Astrophysical Data: Planets 83 Stars, Springer

Law, N. M., Mackay, C. D. \& Baldwin, J. E. 2006, A\&A 446, 739

Popper, D. 1980, ARAA 18, 115

Prieur, J.-L., Koechlin, L., Andre, C., Gallou, G., Lucuix, C. 1998, Experimental Astronomy 8, 297

Scardia, M., Prieur, J.-L., Pansecchi, L., Argyle, R. W., Basso, S., Sala, M., Ghigo, M., Koechlin, L., Aristidi, E. 2006a, MNRAS (submitted)

Scardia, M., Argyle, R. W., Prieur, J.-L., Pansecchi, L., Basso, S., Law, N. M., Mackay, C. D. 2006b, $A N$ (submitted)

Svechnikov, M. A. \& Taidakova, T. A. 1984 Soviet Astronomy 28, 84

Trimble, V. 1974 AJ 79, 967 